\title{
Dark energy, cosmological constant and neutrino mixing
}

\author{
A.Capolupo ${ }^{\natural}$, S.Capozziello ${ }^{\sharp}$, G.Vitiello ${ }^{b}$ \\ Ł Department of Physics and Astronomy, University of Leeds, Leeds LS2 9JT UK, \\ b Dipartimento di Matematica e Informatica, Università di Salerno and Istituto Nazionale di Fisica Nucleare, \\ Gruppo Collegato di Salerno, 84100 Salerno, Italy, \\ \# Dipartimento di Scienze Fisiche, Università di Napoli "Federico II" and INFN Sez. di Napoli, \\ Compl. Univ. Monte S. Angelo, Ed.N, Via Cinthia, I-80126 Napoli, Italy.
}

\begin{abstract}
The today estimated value of dark energy can be achieved by the vacuum condensate induced by neutrino mixing phenomenon. Such a tiny value is recovered for a cut-off of the order of Planck scale and it is linked to the sub-eV neutrino mass scale. Contributions to dark energy from auxiliary fields or mechanisms are not necessary in this approach.
\end{abstract}

PACS numbers: 98.80.Cq, 98.80. Hw, 04.20.Jb, 04.50+h

\section{INTRODUCTION}

The neutrino mixing phenomenon, was firstly studied in the context of quantum mechanics [1, 2, 3, 4, , 5, 6, 7, 8, 9], and more recently analyzed in the framework of the quantum field theory (QFT) formalism [10, 11, 12, 13, 14, 15, $16,17,18,19,20,21$.

The recent experimental achievements proving neutrino oscillations [22, 23] and the progresses in the QFT understanding [20, 21] of the neutrino mixing seems to indicate a promising path beyond the Standard Model of electro-weak interaction for elementary particles and a possible link between high energy physics and cosmology [24, 25]. In this paper, indeed, we show that the energy content of the neutrino mixing vacuum condensate [24] can be interpreted as dynamically evolving dark energy [25] that, at present epoch, assumes the behavior and the value of the observed cosmological constant. We compute such a value and show that, above a threshold, it is slowly diverging and its derivative with respect to the cut-off value goes actually to zero (cfr. Fig.(2)), which allows to use the cut-off at its Planck scale value.

Our result links together dark energy with the sub-eV neutrino mass scale. The link comes from the neutrinoantineutrino pair vacuum condensate due to the mixing phenomenon.

This fact is crucial from a genuine experimental point of view since, up to now, none of the exotic candidates for dark matter and dark energy, has been detected at a fundamental level. Considering neutrino mixing vacuum condensate as the source of dark energy fits with the conservative view by which only actually observed ingredients as gravity, radiation, neutrinos and baryons are taken into account.

The layout of the paper is the following. In Section II, we outline the neutrino mixing formalism in Quantum Field Theory. In Section III we compute the neutrino mixing contribution to the dark energy in the case of two generations. The case of the three flavor fermion mixing is analyzed in Section IV and conclusions are drawn in Section V.

\section{NEUTRINO MIXING IN QUANTUM FIELD THEORY}

For the reader convenience the main features of the QFT formalism for the neutrino mixing are here summarized. For the sake of simplicity, we restrict to the case of two flavors. Extension to three flavors [16] can be also considered (for a detailed review see [19]).

The Pontecorvo mixing transformations for two Dirac neutrino fields are

$$
\begin{aligned}
& \nu_{e}(x)=\nu_{1}(x) \cos \theta+\nu_{2}(x) \sin \theta \\
& \nu_{\mu}(x)=-\nu_{1}(x) \sin \theta+\nu_{2}(x) \cos \theta,
\end{aligned}
$$

where $\nu_{e}(x)$ and $\nu_{\mu}(x)$ are the fields with definite flavors, $\theta$ is the mixing angle and $\nu_{1}$ and $\nu_{2}$ are the fields with definite masses $m_{1} \neq m_{2}$ :

$$
\nu_{i}(x)=\frac{1}{\sqrt{V}} \sum_{\mathbf{k}, r}\left[u_{\mathbf{k}, i}^{r} \alpha_{\mathbf{k}, i}^{r}(t)+v_{-\mathbf{k}, i}^{r} \beta_{-\mathbf{k}, i}^{r \dagger}(t)\right] e^{i \mathbf{k} \cdot \mathbf{x}}, \quad i=1,2,
$$

with $\alpha_{\mathbf{k}, i}^{r}(t)=\alpha_{\mathbf{k}, i}^{r} e^{-i \omega_{k, i} t}, \beta_{\mathbf{k}, i}^{r \dagger}(t)=\beta_{\mathbf{k}, i}^{r \dagger} e^{i \omega_{k, i} t}$, and $\omega_{k, i}=\sqrt{\mathbf{k}^{2}+m_{i}^{2}}$. The operators $\alpha_{\mathbf{k}, i}^{r}$ and $\beta_{\mathbf{k}, i}^{r}, i=1,2, r=1,2$ annihilate the vacuum state $|0\rangle_{1,2} \equiv|0\rangle_{1} \otimes|0\rangle_{2}: \quad \alpha_{\mathbf{k}, i}^{r}|0\rangle_{12}=\beta_{\mathbf{k}, i}^{r}|0\rangle_{12}=0$. The anticommutation relations are: 
$\left\{\nu_{i}^{\alpha}(x), \nu_{j}^{\beta \dagger}(y)\right\}_{t=t^{\prime}}=\delta^{3}(\mathbf{x}-\mathbf{y}) \delta_{\alpha \beta} \delta_{i j}$, with $\alpha, \beta=1, \ldots 4$, and $\left\{\alpha_{\mathbf{k}, i}^{r}, \alpha_{\mathbf{q}, j}^{s \dagger}\right\}=\delta_{\mathbf{k q}} \delta_{r s} \delta_{i j} ;\left\{\beta_{\mathbf{k}, i}^{r}, \beta_{\mathbf{q}, j}^{s \dagger}\right\}=\delta_{\mathbf{k q}} \delta_{r s} \delta_{i j}$, with $i, j=1,2$. All other anticommutators are zero. The orthonormality and completeness relations are: $u_{\mathbf{k}, i}^{r \dagger} u_{\mathbf{k}, i}^{s}=$ $v_{\mathbf{k}, i}^{r \dagger} v_{\mathbf{k}, i}^{s}=\delta_{r s}, \quad u_{\mathbf{k}, i}^{r \dagger} v_{-\mathbf{k}, i}^{s}=v_{-\mathbf{k}, i}^{r \dagger} u_{\mathbf{k}, i}^{s}=0$, and $\sum_{r}\left(u_{\mathbf{k}, i}^{r} u_{\mathbf{k}, i}^{r \dagger}+v_{-\mathbf{k}, i}^{r} v_{-\mathbf{k}, i}^{r \dagger}\right)=1$.

The mixing transformation Eqs. (1) can be written as [10]:

$$
\begin{aligned}
& \nu_{e}^{\alpha}(x)=G_{\theta}^{-1}(t) \nu_{1}^{\alpha}(x) G_{\theta}(t) \\
& \nu_{\mu}^{\alpha}(x)=G_{\theta}^{-1}(t) \nu_{2}^{\alpha}(x) G_{\theta}(t)
\end{aligned}
$$

where the mixing generator $G_{\theta}(t)$ is given by

$$
G_{\theta}(t)=\exp \left[\theta \int d^{3} \mathbf{x}\left(\nu_{1}^{\dagger}(x) \nu_{2}(x)-\nu_{2}^{\dagger}(x) \nu_{1}(x)\right)\right] .
$$

At finite volume, $G_{\theta}(t)$ is an unitary operator, $G_{\theta}^{-1}(t)=G_{-\theta}(t)=G_{\theta}^{\dagger}(t)$, preserving the canonical anticommutation relations; $G_{\theta}^{-1}(t)$ maps the Hilbert spaces for free fields $\mathcal{H}_{1,2}$ to the Hilbert spaces for interacting fields $\mathcal{H}_{e, \mu}: G_{\theta}^{-1}(t)$ : $\mathcal{H}_{1,2} \mapsto \mathcal{H}_{e, \mu}$. In particular, for the vacuum $|0\rangle_{1,2}$ we have, at finite volume $V$ :

$$
|0(t)\rangle_{e, \mu}=G_{\theta}^{-1}(t)|0\rangle_{1,2}
$$

$|0\rangle_{e, \mu}$ is the vacuum for $\mathcal{H}_{e, \mu}$, which we will refer to as the flavor vacuum. In the infinite volume limit the flavor vacuum $|0(t)\rangle_{e, \mu}$ turns out to be unitary inequivalent to the vacuum for the massive neutrinos $|0\rangle_{1,2}[10]$. This can be proved for any number of generations [15]. The non-perturbative nature of the flavored vacuum for the mixed neutrinos is thus revealed.

Due to the linearity of $G_{\theta}(t)$, we can define the flavor annihilators, relative to the fields $\nu_{e}(x)$ and $\nu_{\mu}(x)$ at each time expressed as (we use $(\sigma, i)=(e, 1),(\mu, 2))$ :

$$
\begin{aligned}
\alpha_{\mathbf{k}, \sigma}^{r}(t) & \equiv G_{\theta}^{-1}(t) \alpha_{\mathbf{k}, i}^{r}(t) G_{\theta}(t) \\
\beta_{\mathbf{k}, \sigma}^{r}(t) & \equiv G_{\theta}^{-1}(t) \beta_{\mathbf{k}, i}^{r}(t) G_{\theta}(t) .
\end{aligned}
$$

The flavor fields can be expanded in the same bases as $\nu_{i}$ :

$$
\nu_{\sigma}(\mathbf{x}, t)=\frac{1}{\sqrt{V}} \sum_{\mathbf{k}, r} e^{i \mathbf{k} \cdot \mathbf{x}}\left[u_{\mathbf{k}, i}^{r} \alpha_{\mathbf{k}, \sigma}^{r}(t)+v_{-\mathbf{k}, i}^{r} \beta_{-\mathbf{k}, \sigma}^{r \dagger}(t)\right] .
$$

The flavor annihilation operators in the reference frame such that $\mathbf{k}=(0,0,|\mathbf{k}|)$ are:

$$
\begin{aligned}
\alpha_{\mathbf{k}, e}^{r}(t) & =\cos \theta \alpha_{\mathbf{k}, 1}^{r}(t)+\sin \theta\left(\left|U_{\mathbf{k}}\right| \alpha_{\mathbf{k}, 2}^{r}(t)+\epsilon^{r}\left|V_{\mathbf{k}}\right| \beta_{-\mathbf{k}, 2}^{r \dagger}(t)\right) \\
\alpha_{\mathbf{k}, \mu}^{r}(t) & =\cos \theta \alpha_{\mathbf{k}, 2}^{r}(t)-\sin \theta\left(\left|U_{\mathbf{k}}\right| \alpha_{\mathbf{k}, 1}^{r}(t)-\epsilon^{r}\left|V_{\mathbf{k}}\right| \beta_{-\mathbf{k}, 1}^{r \dagger}(t)\right) \\
\beta_{-\mathbf{k}, e}^{r}(t) & =\cos \theta \beta_{-\mathbf{k}, 1}^{r}(t)+\sin \theta\left(\left|U_{\mathbf{k}}\right| \beta_{-\mathbf{k}, 2}^{r}(t)-\epsilon^{r}\left|V_{\mathbf{k}}\right| \alpha_{\mathbf{k}, 2}^{r \dagger}(t)\right) \\
\beta_{-\mathbf{k}, \mu}^{r}(t) & =\cos \theta \beta_{-\mathbf{k}, 2}^{r}(t)-\sin \theta\left(\left|U_{\mathbf{k}}\right| \beta_{-\mathbf{k}, 1}^{r}(t)+\epsilon^{r}\left|V_{\mathbf{k}}\right| \alpha_{\mathbf{k}, 1}^{r \dagger}(t)\right),
\end{aligned}
$$

with $\epsilon^{r}=(-1)^{r}$ and

$$
\left|U_{\mathbf{k}}\right| \equiv u_{\mathbf{k}, i}^{r \dagger} u_{\mathbf{k}, j}^{r}=v_{-\mathbf{k}, i}^{r \dagger} v_{-\mathbf{k}, j}^{r}, \quad \quad\left|V_{\mathbf{k}}\right| \equiv \epsilon^{r} u_{\mathbf{k}, 1}^{r \dagger} v_{-\mathbf{k}, 2}^{r}=-\epsilon^{r} u_{\mathbf{k}, 2}^{r \dagger} v_{-\mathbf{k}, 1}^{r}
$$

with $i, j=1,2$ and $i \neq j$. We have:

$$
\begin{gathered}
\left|U_{\mathbf{k}}\right|=\left(\frac{\omega_{k, 1}+m_{1}}{2 \omega_{k, 1}}\right)^{\frac{1}{2}}\left(\frac{\omega_{k, 2}+m_{2}}{2 \omega_{k, 2}}\right)^{\frac{1}{2}}\left(1+\frac{\mathbf{k}^{2}}{\left(\omega_{k, 1}+m_{1}\right)\left(\omega_{k, 2}+m_{2}\right)}\right) \\
\left|V_{\mathbf{k}}\right|=\left(\frac{\omega_{k, 1}+m_{1}}{2 \omega_{k, 1}}\right)^{\frac{1}{2}}\left(\frac{\omega_{k, 2}+m_{2}}{2 \omega_{k, 2}}\right)^{\frac{1}{2}}\left(\frac{k}{\left(\omega_{k, 2}+m_{2}\right)}-\frac{k}{\left(\omega_{k, 1}+m_{1}\right)}\right) \\
\left|U_{\mathbf{k}}\right|^{2}+\left|V_{\mathbf{k}}\right|^{2}=1 .
\end{gathered}
$$




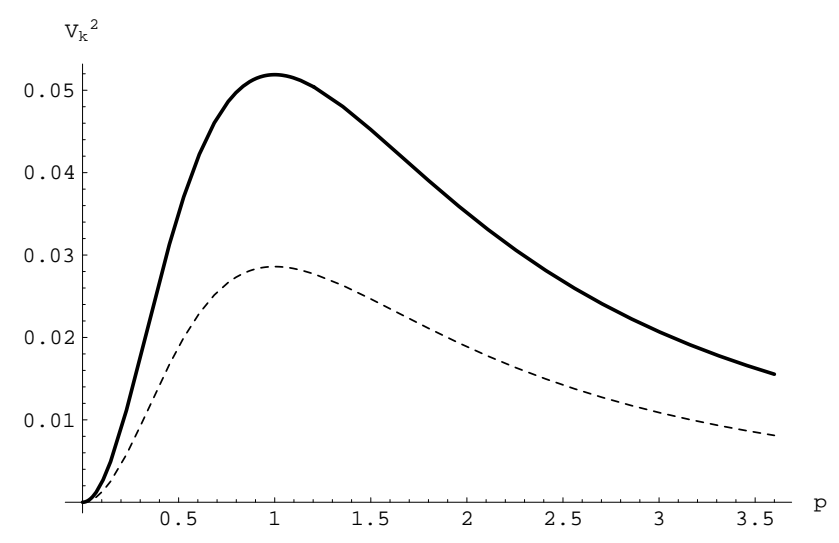

Figure 1: The fermion condensation density $|V(p, a)|^{2}$ as a function of $p$ for $a=0.98$ (solid line) and $a=0.5$ (dashed line).

The condensation density is given by

$$
{ }_{e, \mu}\left\langle 0\left|\alpha_{\mathbf{k}, i}^{r \dagger} \alpha_{\mathbf{k}, i}^{r}\right| 0\right\rangle_{e, \mu}={ }_{e, \mu}\left\langle 0\left|\beta_{\mathbf{k}, i}^{r \dagger} \beta_{\mathbf{k}, i}^{r}\right| 0\right\rangle_{e, \mu}=\sin ^{2} \theta\left|V_{\mathbf{k}}\right|^{2}, \quad i=1,2 .
$$

The Bogoliubov coefficient $\left|V_{\mathbf{k}}\right|^{2}$ appearing in the condensation density can be written as a function of the dimensionless momentum $p=\frac{|\mathbf{k}|}{\sqrt{m_{1} m_{2}}}$ and dimensionless parameter $a=\frac{\left(m_{2}-m_{1}\right)^{2}}{m_{1} m_{2}}$, as follows,

$$
|V(p, a)|^{2}=\frac{1}{2}\left(1-\frac{p^{2}+1}{\sqrt{\left(p^{2}+1\right)^{2}+a p^{2}}}\right) .
$$

From Fig.(1) we see that the effect is maximal when $p=1$, and $|V|^{2}$ goes to zero for large momenta (i.e. for $\left.|\mathbf{k}|^{2} \gg m_{1} m_{2}\right)$ as $|V|^{2} \approx \frac{(\Delta m)^{2}}{4 k^{2}}$.

Since the experimentally observed neutrinos are always extremely relativistic, the value of $|V|^{2}$ is very small. Only for extremely low energies (like those in neutrino cosmological background) $|V|^{2}$ might be large and account for few percent.

In the next Section we will show that the mixing of neutrinos may contribute to the value of the dark energy exactly because of the non-zero value of $\left|V_{\mathbf{k}}\right|^{2}$ : its behavior at very high momenta, together with the Lorentz invariance of the vacuum condensate at the present time, can be responsible of the very tiny value of the cosmological constant.

\section{NEUTRINO MIXING AND DARK ENERGY}

Experimental data indicate that the today observed universe can be described as an accelerating Hubble fluid where the contribution of dark energy component to the total matter-energy density is $\Omega_{\Lambda} \simeq 0.7$ (see the discussion in Section $\mathrm{V}$ ). Moreover, the cosmic flow is "today" accelerating while it was not so at intermediate redshift $z$ (e.g. $1<z<10$ ) where large scale structures have supposed to be clustered. Thus, physically motivated cosmological models should undergo, at least, three phases: an early accelerated inflationary phase, an intermediate standard matter dominated (decelerated) phase and a final, today observed, dark energy dominated (accelerated) phase. This means that we have to take into account some form of dark energy which evolves from early epochs inducing the today observed acceleration.

In this Section we show that the energy density due to neutrino mixing vacuum condensate can be interpreted as an evolving dark energy that at present epoch has a behavior and a value compatible with the observed cosmological constant.

The calculation here presented is performed in a Minkowski space-time but it can be easily extended to curved space-times. When particle mixing and oscillations in curved background is analyzed, neutrino mixing, and in general particle mixing, gives a time dependent dark energy leading, however, to the same final result: the today observed cosmological dark energy value can be recovered.

Let us calculate the contribution $\rho_{v a c}^{\text {mix }}$ of the neutrino mixing to the vacuum energy density.

As well known [26], the Lorentz invariance of the vacuum implies that the vacuum energy-momentum tensor is equal to zero: $\mathcal{T}_{\mu \nu}^{v a c}=\left\langle 0\left|: \mathcal{T}_{\mu \nu}:\right| 0\right\rangle=0$, (as usual normal ordering is denoted by the colon : ... :). The $(0,0)$ component of 
the energy-momentum tensor density $\mathcal{T}_{00}(x)$ for the fields $\nu_{1}$ and $\nu_{2}$ is then

$$
: \mathcal{T}_{00}(x):=\frac{i}{2}:\left(\bar{\Psi}_{m}(x) \gamma_{0} \stackrel{\leftrightarrow}{\partial}_{0} \Psi_{m}(x)\right):
$$

where $\Psi_{m}=\left(\nu_{1}, \nu_{2}\right)$. In terms of the annihilation and creation operators of fields $\nu_{1}$ and $\nu_{2}$, the $(0,0)$ component of the energy-momentum tensor $T_{00}=\int d^{3} x \mathcal{T}_{00}(x)$ is given by

$$
: T_{(i)}^{00}:=\sum_{r} \int d^{3} \mathbf{k} \omega_{k, i}\left(\alpha_{\mathbf{k}, i}^{r \dagger} \alpha_{\mathbf{k}, i}^{r}+\beta_{-\mathbf{k}, i}^{r \dagger} \beta_{-\mathbf{k}, i}^{r}\right)
$$

with $i=1,2$. Note that $T_{(i)}^{00}$ is time independent.

In the early universe epochs, when the Lorentz invariance of the vacuum condensate is broken, $\rho_{v a c}^{m i x}$ presents also space-time dependent condensate contributions. This implies that the contribution $\rho_{v a c}^{\text {mix }}$ of the neutrino mixing to the vacuum energy density is given by computing the expectation value of $T_{(i)}^{00}$ in the flavor vacuum $|0(t)\rangle_{e, \mu}$ :

$$
\rho_{v a c}^{\operatorname{mix}}=\frac{1}{V} \eta_{00 \text { e, }}\left\langle 0(t)\left|\sum_{i}: T_{(i)}^{00}(0):\right| 0(t)\right\rangle_{e, \mu}
$$

Within the QFT formalism for neutrino mixing, we have

$$
{ }_{e, \mu}\left\langle 0\left|: T_{(i)}^{00}:\right| 0\right\rangle_{e, \mu}={ }_{e, \mu}\left\langle 0(t)\left|: T_{(i)}^{00}:\right| 0(t)\right\rangle_{e, \mu}
$$

for any t. We then obtain

$$
\rho_{v a c}^{m i x}=\sum_{i, r} \int \frac{d^{3} \mathbf{k}}{(2 \pi)^{3}} \omega_{k, i}\left({ }_{e, \mu}\left\langle 0\left|\alpha_{\mathbf{k}, i}^{r \dagger} \alpha_{\mathbf{k}, i}^{r}\right| 0\right\rangle_{e, \mu}+{ }_{e, \mu}\left\langle 0\left|\beta_{\mathbf{k}, i}^{r \dagger} \beta_{\mathbf{k}, i}^{r}\right| 0\right\rangle_{e, \mu}\right),
$$

and then

$$
\rho_{v a c}^{\operatorname{mix}}=4 \sin ^{2} \theta \int \frac{d^{3} \mathbf{k}}{(2 \pi)^{3}}\left(\omega_{k, 1}+\omega_{k, 2}\right)\left|V_{\mathbf{k}}\right|^{2},
$$

which, introducing the cut-off $K$, becomes

$$
\rho_{v a c}^{\operatorname{mix}}=\frac{2}{\pi} \sin ^{2} \theta \int_{0}^{K} d k k^{2}\left(\omega_{k, 1}+\omega_{k, 2}\right)\left|V_{\mathbf{k}}\right|^{2} .
$$

In a similar way, the contribution $p_{v a c}^{\text {mix }}$ of the neutrino mixing to the vacuum pressure is given by the expectation value of $T_{(i)}^{j j}$ (where no summation on the index $j$ is intended) on the flavor vacuum $|0\rangle_{e, \mu}$ :

$$
p_{v a c}^{m i x}=-\frac{1}{V} \eta_{j j e, \mu}\left\langle 0\left|\sum_{i}: T_{(i)}^{j j}:\right| 0\right\rangle_{e, \mu}
$$

Being

$$
: T_{(i)}^{j j}:=\sum_{r} \int d^{3} \mathbf{k} \frac{k^{j} k^{j}}{\omega_{k, i}}\left(\alpha_{\mathbf{k}, i}^{r \dagger} \alpha_{\mathbf{k}, i}^{r}+\beta_{-\mathbf{k}, i}^{r \dagger} \beta_{-\mathbf{k}, i}^{r}\right)
$$

in the case of the isotropy of the momenta we have $T^{11}=T^{22}=T^{33}$, then

$$
p_{v a c}^{\text {mix }}=\frac{2}{3 \pi} \sin ^{2} \theta \int_{0}^{K} d k k^{4}\left[\frac{1}{\omega_{k, 1}}+\frac{1}{\omega_{k, 2}}\right]\left|V_{\mathbf{k}}\right|^{2} .
$$

From Eqs.(19) and (22) we have that the adiabatic index is $w=p_{v a c}^{\text {mix }} / \rho_{\text {vac }}^{\text {mix }} \simeq 1 / 3$ when the cut-off is chosen to be $K \gg m_{1}, m_{2}$.

The values of $\rho_{v a c}^{\text {mix }}$ and $p_{v a c}^{\text {mix }}$ which we obtain are time-independent since we are taking into account the Minkowski metric. Considering a curved space-time, time-dependence has to be taken into account but the essence of the result 
is the same. At the present epoch, the breaking of the Lorentz invariance is negligible and then $\rho_{v a c}^{m i x}$ comes from space-time independent condensate contributions (i.e. the contributions carrying a non-vanishing $\partial_{\mu} \sim k_{\mu}=\left(\omega_{k}, k_{j}\right)$ are missing). That is, the energy-momentum density tensor of the vacuum condensate is given by

$$
{ }_{e, \mu}\left\langle 0\left|: T_{\mu \nu}:\right| 0\right\rangle_{e, \mu}=\eta_{\mu \nu} \sum_{i} m_{i} \int \frac{d^{3} x}{(2 \pi)^{3}}{ }_{e, \mu}\left\langle 0\left|: \bar{\nu}_{i}(x) \nu_{i}(x):\right| 0\right\rangle_{e, \mu}=\eta_{\mu \nu} \rho_{\Lambda}^{m i x} .
$$

Since $\eta_{\mu \nu}=\operatorname{diag}(1,-1,-1,-1)$ and, in a homogeneous and isotropic universe, the energy-momentum tensor is $T_{\mu \nu}=\operatorname{diag}(\rho, p, p, p)$, then, consistently with Lorentz invariance, the state equation is $\rho_{\Lambda}^{m i x}=-p_{\Lambda}^{m i x}$. This means that the vacuum condensate, coming from neutrino mixing, contributes today to the dynamics of the universe by a cosmological constant behavior [25]. Explicitly, we have

$$
\rho_{\Lambda}^{m i x}=\frac{2}{\pi} \sin ^{2} \theta \int_{0}^{K} d k k^{2}\left[\frac{m_{1}^{2}}{\omega_{k, 1}}+\frac{m_{2}^{2}}{\omega_{k, 2}}\right]\left|V_{\mathbf{k}}\right|^{2}
$$

Solving the integral, we obtain

$$
\begin{aligned}
\rho_{\Lambda}^{m i x} & =\frac{2}{\pi} \sin ^{2} \theta\left\{\left(m_{2}^{2}-m_{1}^{2}\right) k\left(\sqrt{k^{2}+m_{2}^{2}}-\sqrt{k^{2}+m_{1}^{2}}\right)+\frac{2\left(m_{2}-m_{1}\right)}{\sqrt{m_{2}^{2}-m_{1}^{2}}}\left[m_{1}^{4} \arctan \left(\frac{\sqrt{m_{2}^{2}-m_{1}^{2}}}{m_{1} \sqrt{k^{2}+m_{2}^{2}}} k\right)\right.\right. \\
& \left.-m_{2}^{4} \arctan \left(\frac{\sqrt{m_{2}^{2}-m_{1}^{2}}}{m_{2} \sqrt{k^{2}+m_{1}^{2}}} k\right)\right]+\left(2 m_{1}^{4}-2 m_{1}^{3} m_{2}+m_{1}^{2} m_{2}^{2}-m_{2}^{4}\right) \log \left(k+\sqrt{k^{2}+m_{2}^{2}}\right) \\
& \left.+\left(2 m_{2}^{4}-2 m_{2}^{3} m_{1}+m_{1}^{2} m_{2}^{2}-m_{1}^{4}\right) \log \left(k+\sqrt{k^{2}+m_{1}^{2}}\right)\right\}_{0}^{K},
\end{aligned}
$$

that is

$$
\begin{aligned}
\rho_{\Lambda}^{m i x} & =\frac{2}{\pi} \sin ^{2} \theta\left\{\left(m_{2}^{2}-m_{1}^{2}\right) K\left(\sqrt{K^{2}+m_{2}^{2}}-\sqrt{K^{2}+m_{1}^{2}}\right)+\frac{2\left(m_{2}-m_{1}\right)}{\sqrt{m_{2}^{2}-m_{1}^{2}}}\left[m_{1}^{4} \arctan \left(\frac{\sqrt{m_{2}^{2}-m_{1}^{2}}}{m_{1} \sqrt{K^{2}+m_{2}^{2}}} K\right)\right.\right. \\
& \left.-m_{2}^{4} \arctan \left(\frac{\sqrt{m_{2}^{2}-m_{1}^{2}}}{m_{2} \sqrt{K^{2}+m_{1}^{2}}} K\right)\right]+\left(2 m_{1}^{4}-2 m_{1}^{3} m_{2}+m_{1}^{2} m_{2}^{2}-m_{2}^{4}\right) \log \left(K+\sqrt{K^{2}+m_{2}^{2}}\right) \\
& +\left(2 m_{2}^{4}-2 m_{2}^{3} m_{1}+m_{1}^{2} m_{2}^{2}-m_{1}^{4}\right) \log \left(K+\sqrt{K^{2}+m_{1}^{2}}\right)-\left(2 m_{1}^{4}-2 m_{1}^{3} m_{2}+m_{1}^{2} m_{2}^{2}-m_{2}^{4}\right) \log \left(m_{2}\right) \\
& \left.-\left(2 m_{2}^{4}-2 m_{2}^{3} m_{1}+m_{1}^{2} m_{2}^{2}-m_{1}^{4}\right) \log \left(m_{1}\right)\right\} .
\end{aligned}
$$

The plot of $\rho_{\Lambda}^{m i x}$ as function of the momentum cut-off $K$ (Fig.2) shows that for $K$ at Planck scale a value of $\rho_{\Lambda}^{\text {mix }}$ is obtained, which is in agreement with the observed value of cosmological constant.

To better understand the meaning of Eq.(26), we report the behavior of $\rho_{\Lambda}^{\operatorname{mix}}$ for $K \gg m_{1}, m_{2}$ :

$$
\begin{aligned}
\rho_{\Lambda}^{m i x} & \approx \frac{2}{\pi} \sin ^{2} \theta\left\{\frac{2\left(m_{2}-m_{1}\right)}{\sqrt{m_{2}^{2}-m_{1}^{2}}}\left[m_{1}^{4} \arctan \left(\frac{\sqrt{m_{2}^{2}-m_{1}^{2}}}{m_{1}}\right)-m_{2}^{4} \arctan \left(\frac{\sqrt{m_{2}^{2}-m_{1}^{2}}}{m_{2}}\right)\right]\right. \\
& -\left(2 m_{1}^{4}-2 m_{1}^{3} m_{2}+m_{1}^{2} m_{2}^{2}-m_{2}^{4}\right) \log \left(m_{2}\right)-\left(2 m_{2}^{4}-2 m_{2}^{3} m_{1}+m_{1}^{2} m_{2}^{2}-m_{1}^{4}\right) \log \left(m_{1}\right) \\
& \left.+\left(m_{1}^{4}+m_{2}^{4}+2 m_{1}^{2} m_{2}^{2}-2 m_{1}^{3} m_{2}-2 m_{2}^{3} m_{1}\right) \log (2 K)\right\} .
\end{aligned}
$$

This shows that the integral diverges in $K$ as $m_{i}^{4} \log (K)$. As shown in Fig.2 the divergence in $K$ is smoothed by the factor $m_{i}^{4}$. For neutrino masses of order of $10^{-3} \mathrm{eV}$ we have $\rho_{\Lambda}^{\operatorname{mix}}=5.4 \times 10^{-47} \mathrm{GeV}^{4}$ for a value of the cut-off of order of the Planck scale $K=10^{19} \mathrm{GeV}$. From Eq.(27) one also sees that $\frac{d \rho_{\Lambda}^{\operatorname{mix}}(K)}{d K} \propto \frac{1}{K} \rightarrow 0$ for large $K$. An interesting question to ask is how the result $\rho_{\Lambda}^{\text {mix }} \propto m_{i}^{4} \log (K)$, directly obtained in our approach, is related to the conjecture [27] that the small value of the cosmological constant $\rho_{\Lambda} \propto\left(10^{-3} \mathrm{eV}\right)^{4}$ is associated with the vacuum in a theory which has a fundamental mass scale $m \sim 10^{-3} \mathrm{eV}$.

We observe that, since, at present epoch, the characteristic oscillation length of the neutrino is much smaller than the universe curvature radius, the mixing treatment in the flat space-time, in such an epoch, is a good approximation of that in FRW space-time. The central result of this paper is: the vacuum condensate from neutrino mixing can give rise to the observed value of the cosmological constant. Exotic components to dark energy are not necessary in this approach. 


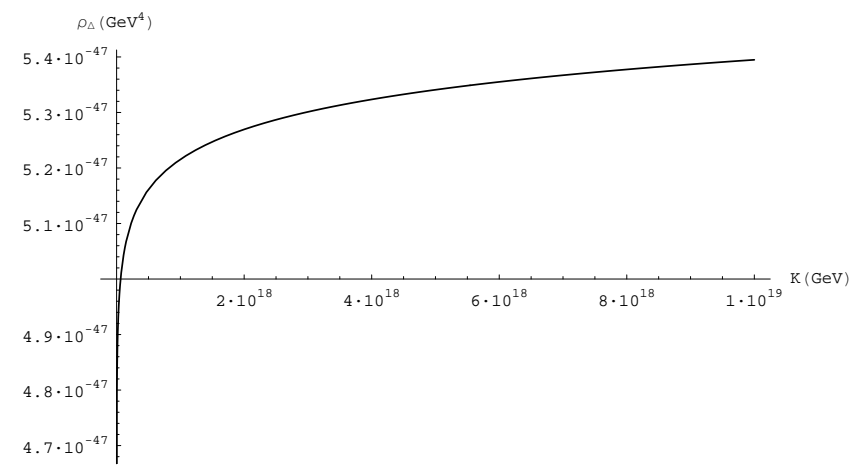

Figure 2: The neutrino mixing dark energy as a function of cut-off K.

\section{THREE FLAVOR FERMION MIXING}

The above result can be easily extended to the three flavor fermion mixing case. The Lagrangian density describing three Dirac fields with a mixed mass term is:

$$
\mathcal{L}(x)=\bar{\Psi}_{f}(x)(i \not \partial-\mathrm{M}) \Psi_{f}(x),
$$

where $\Psi_{f}^{T}=\left(\nu_{e}, \nu_{\mu}, \nu_{\tau}\right)$ and $\mathbf{M}=\mathbf{M}^{\dagger}$ is the mixed mass matrix. Among the various possible parameterizations of the mixing matrix for three fields, we work with CKM matrix of the form:

$$
\Psi_{f}(x)=\mathcal{U} \Psi_{m}(x)=\left(\begin{array}{ccc}
c_{12} c_{13} & s_{12} c_{13} & s_{13} e^{-i \delta} \\
-s_{12} c_{23}-c_{12} s_{23} s_{13} e^{i \delta} & c_{12} c_{23}-s_{12} s_{23} s_{13} e^{i \delta} & s_{23} c_{13} \\
s_{12} s_{23}-c_{12} c_{23} s_{13} e^{i \delta} & -c_{12} s_{23}-s_{12} c_{23} s_{13} e^{i \delta} & c_{23} c_{13}
\end{array}\right) \Psi_{m}(x)
$$

with $c_{i j}=\cos \theta_{i j}$ and $s_{i j}=\sin \theta_{i j}$, being $\theta_{i j}$ the mixing angle between $\nu_{i}, \nu_{j}$ and $\Psi_{m}^{T}=\left(\nu_{1}, \nu_{2}, \nu_{3}\right)$. Using Eq. (29), we diagonalize the quadratic form of Eq.(28), which then reduces to the Lagrangian for three Dirac fields, with masses $m_{1}, m_{2}$ and $m_{3}$ :

$$
\mathcal{L}(x)=\bar{\Psi}_{m}(x)\left(i \not \partial-\mathrm{M}_{d}\right) \Psi_{m}(x),
$$

where $\mathrm{M}_{d}=\operatorname{diag}\left(m_{1}, m_{2}, m_{3}\right)$.

The mixing transformation can be written as $\nu_{\sigma}^{\alpha}(x) \equiv G_{\theta}^{-1}(t) \nu_{i}^{\alpha}(x) G_{\theta}(t)$, where $(\sigma, i)=(e, 1),(\mu, 2),(\tau, 3)$, and the generator is now

$$
G_{\theta}(t)=G_{23}(t) G_{13}(t) G_{12}(t)
$$

where

$$
\begin{aligned}
G_{12}(t) & \equiv \exp \left[\theta_{12} \int d^{3} x\left(\nu_{1}^{\dagger}(x) \nu_{2}(x)-\nu_{2}^{\dagger}(x) \nu_{1}(x)\right)\right] \\
G_{23}(t) & \equiv \exp \left[\theta_{23} \int d^{3} x\left(\nu_{2}^{\dagger}(x) \nu_{3}(x)-\nu_{3}^{\dagger}(x) \nu_{2}(x)\right)\right] \\
G_{13}(t) & \equiv \exp \left[\theta_{13} \int d^{3} x\left(\nu_{1}^{\dagger}(x) \nu_{3}(x) e^{-i \delta}-\nu_{3}^{\dagger}(x) \nu_{1}(x) e^{i \delta}\right)\right] .
\end{aligned}
$$

The vacuum for the mass eigenstates is denoted by $|0\rangle_{m}$ and the flavor vacuum is given by $|0(t)\rangle_{f} \equiv G_{\theta}^{-1}(t)|0\rangle_{m}$. 
The flavor annihilation operators in the reference frame $\mathbf{k}=(0,0,|\mathbf{k}|)$ are:

$$
\begin{aligned}
\alpha_{\mathbf{k}, e}^{r}(t)= & c_{12} c_{13} \alpha_{\mathbf{k}, 1}^{r}(t)+s_{12} c_{13}\left(\left|U_{12}^{\mathbf{k}}\right| \alpha_{\mathbf{k}, 2}^{r}(t)+\epsilon^{r}\left|V_{12}^{\mathbf{k}}\right| \beta_{-\mathbf{k}, 2}^{r \dagger}(t)\right)+e^{-i \delta} s_{13}\left(\left|U_{13}^{\mathbf{k}}\right| \alpha_{\mathbf{k}, 3}^{r}(t)+\epsilon^{r}\left|V_{13}^{\mathbf{k}}\right| \beta_{-\mathbf{k}, 3}^{r \dagger}(t)\right)(35) \\
\alpha_{\mathbf{k}, \mu}^{r}(t)= & \left(c_{12} c_{23}-e^{i \delta} s_{12} s_{23} s_{13}\right) \alpha_{\mathbf{k}, 2}^{r}(t)-\left(s_{12} c_{23}+e^{i \delta} c_{12} s_{23} s_{13}\right)\left(\left|U_{12}^{\mathbf{k}}\right| \alpha_{\mathbf{k}, 1}^{r}(t)-\epsilon^{r}\left|V_{12}^{\mathbf{k}}\right| \beta_{-\mathbf{k}, 1}^{r \dagger}(t)\right) \\
& +s_{23} c_{13}\left(\left|U_{23}^{\mathbf{k}}\right| \alpha_{\mathbf{k}, 3}^{r}(t)+\epsilon^{r}\left|V_{23}^{\mathbf{k}}\right| \beta_{-\mathbf{k}, 3}^{r \dagger}(t)\right) \\
\alpha_{\mathbf{k}, \tau}^{r}(t)= & c_{23} c_{13} \alpha_{\mathbf{k}, 3}^{r}(t)-\left(c_{12} s_{23}+e^{i \delta} s_{12} c_{23} s_{13}\right)\left(\left|U_{23}^{\mathbf{k}}\right| \alpha_{\mathbf{k}, 2}^{r}(t)-\epsilon^{r}\left|V_{23}^{\mathbf{k}}\right| \beta_{-\mathbf{k}, 2}^{r \dagger}(t)\right) \\
& +\left(s_{12} s_{23}-e^{i \delta} c_{12} c_{23} s_{13}\right)\left(\left|U_{13}^{\mathbf{k}}\right| \alpha_{\mathbf{k}, 1}^{r}(t)-\epsilon^{r}\left|V_{13}^{\mathbf{k}}\right| \beta_{-\mathbf{k}, 1}^{r \dagger}(t)\right), \\
\beta_{-\mathbf{k}, e}^{r}(t)= & c_{12} c_{13} \beta_{-\mathbf{k}, 1}^{r}(t)+s_{12} c_{13}\left(\left|U_{12}^{\mathbf{k}}\right| \beta_{-\mathbf{k}, 2}^{r}(t)-\epsilon^{r}\left|V_{12}^{\mathbf{k}}\right| \alpha_{\mathbf{k}, 2}^{r \dagger}(t)\right)+e^{i \delta} s_{13}\left(\left|U_{13}^{\mathbf{k}}\right| \beta_{-\mathbf{k}, 3}^{r}(t)-\epsilon^{r}\left|V_{13}^{\mathbf{k}}\right| \alpha_{\mathbf{k}, 3}^{r \dagger}(t)\right)(38) \\
\beta_{-\mathbf{k}, \mu}^{r}(t)= & \left(c_{12} c_{23}-e^{-i \delta} s_{12} s_{23} s_{13}\right) \beta_{-\mathbf{k}, 2}^{r}(t)-\left(s_{12} c_{23}+e^{-i \delta} c_{12} s_{23} s_{13}\right)\left(\left|U_{12}^{\mathbf{k}}\right| \beta_{-\mathbf{k}, 1}^{r}(t)+\epsilon^{r}\left|V_{12}^{\mathbf{k}}\right| \alpha_{\mathbf{k}, 1}^{r \dagger}(t)\right)+ \\
& +s_{23} c_{13}\left(\left|U_{23}^{\mathbf{k}}\right| \beta_{-\mathbf{k}, 3}^{r}(t)-\epsilon^{r}\left|V_{23}^{\mathbf{k}}\right| \alpha_{\mathbf{k}, 3}^{r \dagger}(t)\right), \\
& +\left(s_{12} s_{23}-e^{-i \delta} c_{12} c_{23} s_{13}\right)\left(\left|U_{13}^{\mathbf{k}}\right| \beta_{-\mathbf{k}, 1}^{r}(t)+\epsilon^{r}\left|V_{13}^{\mathbf{k}}\right| \alpha_{\mathbf{k}, 1}^{r \dagger}(t)\right) \cdot \\
\beta_{-\mathbf{k}, \tau}^{r}(t)= & c_{23} c_{13} \beta_{-\mathbf{k}, 3}^{r}-\left(c_{12} s_{23}+e^{-i \delta} s_{12} c_{23} s_{13}\right)\left(\left|U_{23}^{\mathbf{k}}\right| \beta_{-\mathbf{k}, 2}^{r}(t)+\epsilon^{r}\left|V_{23}^{\mathbf{k}}\right| \alpha_{\mathbf{k}, 2}^{r \dagger}(t)\right) \\
& \\
& (39)
\end{aligned}
$$

These operators satisfy canonical (anti)commutation relations at equal times. $U_{i j}^{\mathbf{k}}$ and $V_{i j}^{\mathbf{k}}$ are Bogoliubov coefficients defined as:

$$
\begin{gathered}
\left|U_{i j}^{\mathbf{k}}\right|=\left(\frac{\omega_{k, i}+m_{i}}{2 \omega_{k, i}}\right)^{\frac{1}{2}}\left(\frac{\omega_{k, j}+m_{j}}{2 \omega_{k, j}}\right)^{\frac{1}{2}}\left(1+\frac{|\mathbf{k}|^{2}}{\left(\omega_{k, i}+m_{i}\right)\left(\omega_{k, j}+m_{j}\right)}\right) \\
\left|V_{i j}^{\mathbf{k}}\right|=\left(\frac{\omega_{k, i}+m_{i}}{2 \omega_{k, i}}\right)^{\frac{1}{2}}\left(\frac{\omega_{k, j}+m_{j}}{2 \omega_{k, j}}\right)^{\frac{1}{2}}\left(\frac{|\mathbf{k}|}{\left(\omega_{k, j}+m_{j}\right)}-\frac{|\mathbf{k}|}{\left(\omega_{k, i}+m_{i}\right)}\right) \\
\left|U_{i j}^{\mathbf{k}}\right|^{2}+\left|V_{i j}^{\mathbf{k}}\right|^{2}=1
\end{gathered}
$$

where $i, j=1,2,3$ and $j>i$. The condensation densities are different for particles of different masses:

$$
\begin{aligned}
& \mathcal{N}_{1}^{\mathbf{k}}={ }_{f}\left\langle 0(t)\left|N_{\alpha_{1}}^{\mathbf{k}, r}\right| 0(t)\right\rangle_{f}={ }_{f}\left\langle 0(t)\left|N_{\beta_{1}}^{\mathbf{k}, r}\right| 0(t)\right\rangle_{f}=s_{12}^{2} c_{13}^{2}\left|V_{12}^{\mathbf{k}}\right|^{2}+s_{13}^{2}\left|V_{13}^{\mathbf{k}}\right|^{2}, \\
& \mathcal{N}_{2}^{\mathbf{k}}={ }_{f}\left\langle 0(t)\left|N_{\alpha_{2}}^{\mathbf{k}, r}\right| 0(t)\right\rangle_{f}={ }_{f}\left\langle 0(t)\left|N_{\beta_{2}}^{\mathbf{k}, r}\right| 0(t)\right\rangle_{f}=\left|-s_{12} c_{23}+e^{i \delta} c_{12} s_{23} s_{13}\right|^{2}\left|V_{12}^{\mathbf{k}}\right|^{2}+s_{23}^{2} c_{13}^{2}\left|V_{23}^{\mathbf{k}}\right|^{2}, \\
& \mathcal{N}_{3}^{\mathbf{k}}={ }_{f}\left\langle 0(t)\left|N_{\alpha_{3}}^{\mathbf{k}, r}\right| 0(t)\right\rangle_{f}={ }_{f}\left\langle 0(t)\left|N_{\beta_{3}}^{\mathbf{k}, r}\right| 0(t)\right\rangle_{f}=\left|-c_{12} s_{23}+e^{i \delta} s_{12} c_{23} s_{13}\right|^{2}\left|V_{23}^{\mathbf{k}}\right|^{2}+\left|s_{12} s_{23}+e^{i \delta} c_{12} c_{23} s_{13}\right|^{2} \mid V_{13}^{\mathbf{k}}\left(\left.\right|^{Z} 6\right) \\
& \rho_{\Lambda}^{m i x}=\frac{2}{\pi} \int_{0}^{K} d k k^{2}\left[\frac{m_{1}^{2}}{\omega_{k, 1}}\left(s_{12}^{2} c_{13}^{2}\left|V_{12}^{\mathbf{k}}\right|^{2}+s_{13}^{2}\left|V_{13}^{\mathbf{k}}\right|^{2}\right)+\frac{m_{2}^{2}}{\omega_{k, 2}}\left(\left|-s_{12} c_{23}+e^{i \delta} c_{12} s_{23} s_{13}\right|^{2}\left|V_{12}^{\mathbf{k}}\right|^{2}+s_{23}^{2} c_{13}^{2}\left|V_{23}^{\mathbf{k}}\right|^{2}\right)\right. \\
& \left.+\frac{m_{3}^{2}}{\omega_{k, 3}}\left(\left|-c_{12} s_{23}+e^{i \delta} s_{12} c_{23} s_{13}\right|^{2}\left|V_{23}^{\mathbf{k}}\right|^{2}+\left|s_{12} s_{23}+e^{i \delta} c_{12} c_{23} s_{13}\right|^{2}\left|V_{13}^{\mathbf{k}}\right|^{2}\right)\right]
\end{aligned}
$$

which can be written as

$$
\begin{aligned}
\rho_{\Lambda}^{m i x} & =\frac{2}{\pi} \int_{0}^{K} d k k^{2}\left\{\frac{m_{1}^{2}}{\omega_{k, 1}}\left(s_{12}^{2} c_{13}^{2}\left|V_{12}^{\mathbf{k}}\right|^{2}+s_{13}^{2}\left|V_{13}^{\mathbf{k}}\right|^{2}\right)+\frac{m_{2}^{2}}{\omega_{k, 2}}\left[\left(s_{12}^{2} c_{23}^{2}+c_{12}^{2} s_{23}^{2} s_{13}^{2}\right)\left|V_{12}^{\mathbf{k}}\right|^{2}+s_{23}^{2} c_{13}^{2}\left|V_{23}^{\mathbf{k}}\right|^{2}\right]\right. \\
& \left.+\frac{m_{3}^{2}}{\omega_{k, 3}}\left[\left(c_{12}^{2} s_{23}^{2}+s_{12}^{2} c_{23}^{2} s_{13}^{2}\right)\left|V_{23}^{\mathbf{k}}\right|^{2}+\left(s_{12}^{2} s_{23}^{2}+c_{12}^{2} c_{23}^{2} s_{13}^{2}\right)\left|V_{13}^{\mathbf{k}}\right|^{2}\right]\right\} \\
& -\frac{4}{\pi} s_{12} c_{23} c_{12} s_{23} s_{13} c_{\delta} \int_{0}^{K} d k k^{2}\left\{\frac{m_{2}^{2}}{\omega_{k, 2}}\left|V_{12}^{\mathbf{k}}\right|^{2}+\frac{m_{3}^{2}}{\omega_{k, 3}}\left[\left|V_{23}^{\mathbf{k}}\right|^{2}-\left|V_{13}^{\mathbf{k}}\right|^{2}\right]\right\}
\end{aligned}
$$


where $c_{\delta}=\cos \delta$. We note that $\rho_{\Lambda}^{m i x}$ is also depending on the $C P$ violating phase $\delta$. Like in the case of two flavor neutrino mixing, the integral diverges in $K$ as $m_{i}^{4} \log (K)$. A value of $\rho_{\Lambda}^{\text {mix }}$, compatible with the upper bound on the cosmological dark energy, is obtained for neutrino masses of order of $10^{-3} \mathrm{eV}$ so the result is essentially the same of the two flavor case.

\section{CONCLUSIONS AND DISCUSSION}

The vacuum condensate generated by neutrino mixing can be interpreted as an evolving dark energy that, at present epoch, behaves as the cosmological constant, giving rise to its observed value. The result is naturally achieved even when a cut-off $K$ of the order of Planck scale is considered. It is easily recovered also for three flavor fermion mixing. Such a result links together dark energy with the neutrino masses. Introducing auxiliary fields or mechanisms is not required in our approach.

A short summary of the observational status of art can aid to clarify the frame for our considerations and results. An increasing bulk of data have been accumulated in the last few years. They have paved the way to the emergence of a new standard cosmological model usually referred to as the concordance model. The Hubble diagram of Type Ia Supernovae (SNeIa), measured by both the Supernova Cosmology Project [28] and the High-z Team [29] up to redshift $z \sim 1$, was the first evidence that the universe is undergoing a phase of accelerated expansion. On the other hand, balloon born experiments, such as BOOMERanG [30] and MAXIMA [31], determined the location of the first and second peak in the anisotropy spectrum of cosmic microwave background radiation (CMBR) pointing out that the geometry of the universe is spatially flat. If combined with constraints coming from galaxy clusters on the matter density parameter $\Omega_{M}$, these data indicate that the universe is dominated by a non-clustered fluid with negative pressure, generically dubbed dark energy, which is able to drive the accelerated expansion. This picture has been further strengthened by the more precise measurements of the CMBR spectrum, due to the WMAP experiment [32], and by the extension of the SNeIa Hubble diagram to redshifts higher than 1 [33]. Several models trying to explain this phenomenon have been presented; the simplest explanation is claiming for the well known cosmological constant $\Lambda$ [34]. Although the best fit to most of the available astrophysical data [32], the $\Lambda$ CDM model fails in explaining why the inferred value of $\Lambda$ is so tiny (120 orders of magnitude lower) compared to the typical vacuum energy values predicted by particle physics and why its energy density is today comparable to the matter density (the so called coincidence problem). As a tentative solution, many authors have replaced the cosmological constant with a scalar field rolling down its potential and giving rise to models referred to as quintessence [35]. Even if successful in fitting the data, the quintessence approach to dark energy is still plagued by the coincidence problem since the dark energy and matter densities evolve differently and reach comparable values for a very limited portion of the universe evolution coinciding at present era. In this case, the coincidence problem is replaced with a fine-tuning problem. Moreover, it is not clear where this scalar field originates from, thus leaving a great uncertainty on the choice of the scalar field potential.

The subtle and elusive nature of dark energy has led to look for completely different scenarios able to give a quintessential behavior without the need of exotic components. To this aim, we observe that the acceleration of the universe only claims for a negative pressure dominant component, but does not tell anything about the nature and the number of cosmic fluids filling the universe [36]. This consideration suggests that it could be possible to explain the accelerated expansion by introducing a single cosmic fluid with an equation of state causing it to act like dark matter at high densities (giving rise to clustered structures) and dark energy at low densities (then giving rise to accelerated behavior of cosmic fluid). An attractive feature of these models, usually referred to as Unified Dark Energy (UDE) or Unified Dark Matter (UDM) models, is that such an approach naturally solves, at least phenomenologically, the coincidence problem. Some interesting examples are the generalized Chaplygin gas [37], the tachyon field [38] and the condensate cosmology [39]. A different class of UDE models has been proposed [40] where a single fluid is considered whose energy density scales with the redshift in such a way that the radiation dominated era, the matter dominated era and the accelerating phase can be naturally achieved. Actually, there is still a different way to face the problem of cosmic acceleration. It is possible that the observed acceleration is not the manifestation of another ingredient in the cosmic pie, but rather the first signal of a breakdown of our understanding of the laws of gravitation [41, 42] . Examples of models comprising only the standard matter are provided by the Cardassian expansion [43], the DGP gravity [44], higher order gravity actions [45], non - vanishing torsion field [46], higher-order curvature invariants included in the gravity Lagrangian [47], etc..

This abundance of models is from one hand the signal of the fact that we have a limited number of cosmological tests to discriminate among rival theories, and from the other hand, that a urgent degeneracy problem has to be faced. The evidences of neutrino oscillations [22, 23] and the fact that the vacuum condensate originated by neutrino mixing provides contributions to the dark energy compatible with today expected value, as shown in the present paper, could contribute towards a solution of such a problem from both experimental and theoretical viewpoints. 


\section{Acknowledgements}

One of the authors (A.C.) acknowledges the Department of Physics and Astronomy, University of Leeds for partial financial support. Support from INFN and MURST is also acknowledged.

[1] B. Pontecorvo, Sov. Phys. JETP 6, 429 (1957) [Zh. Eksp. Teor. Fiz. 33, 549 (1957)].

[2] Z. Maki, M. Nakagawa and S. Sakata, Prog. Theor. Phys. 28, 870 (1962).

[3] K.Fujii, Nuovo Cim. 34, 722 (1964).

[4] V. N. Gribov and B. Pontecorvo, Phys. Lett. B 28, 493 (1969).

[5] S. M. Bilenky and B. Pontecorvo, Phys. Rept. 41, 225 (1978).

[6] S.M. Bilenky and S.T. Petcov, Rev. Mod. Phys. 59, 671 (1987).

T. Cheng and L. Li, Gauge Theory of Elementary Particle Physics, Clarendon Press, Oxford, (1989).

[7] R. N. Mohapatra and P. B. Pal, World Sci. Lect. Notes Phys. 41, 1 (1991).

J.N. Bahcall, "Neutrino Astrophysics", Cambridge Univ. Press, Cambridge, UK, (1989).

[8] L. Wolfenstein, Phys. Rev. D 17, 2369 (1978).

S.P. Mikheev and A. Yu. Smirnov, Sov. J. Nucl. Phys. 42, 913 (1985).

[9] C. Giunti, C. W. Kim and U. W. Lee, Phys. Rev. D 44, 3635 (1991).

[10] M. Blasone and G. Vitiello, Annals Phys. 244, 283 (1995).

[11] M. Blasone, P.A. Henning and G. Vitiello, Phys. Lett. B 451, 140 (1999).

[12] K. Fujii, C. Habe and T. Yabuki, Phys. Rev. D 59, 113003 (1999); Phys. Rev. D 64, 013011 (2001).

[13] C.R. Ji, Y. Mishchenko, Phys. Rev. D 64, 076004 (2001).

[14] C.R. Ji, Y. Mishchenko, Phys. Rev. D 65, 096015 (2002).

[15] K. C. Hannabuss and D. C. Latimer, J. Phys. A 33, 1369 (2000); J. Phys. A 36, L69 (2003).

[16] M. Blasone, A. Capolupo and G. Vitiello, Phys. Rev. D 66, 025033 (2002).

[17] M. Blasone, A. Capolupo, O. Romei and G. Vitiello, Phys. Rev. D 63, 125015 (2001).

[18] A. Capolupo, C. R. Ji, Y. Mishchenko and G. Vitiello, Phys. Lett. B 594, 135 (2004).

[19] A. Capolupo, Ph.D. Thesis hep-th/0408228.

[20] M. Blasone, A. Capolupo, F. Terranova and G. Vitiello, Phys. Rev. D 72, 013003 (2005).

M. Blasone, A. Capolupo and G. Vitiello, Acta Phys. Polon. B 36, 3245 (2005).

[21] M. Blasone, A. Capolupo, C. R. Ji and G. Vitiello, arXiv:hep-ph/0611106.

[22] SNO Collaboration, Phys. Rev. Lett. 89, No. 1, 011301 (2002).

[23] K2K collaboration, E. Aliu et al, Phys. Rev. Lett. 94, 081802 (2005).

[24] M. Blasone, A. Capolupo, S. Capozziello, S. Carloni and G. Vitiello, Phys. Lett. A 323, 182 (2004).

[25] A. Capolupo, S. Capozziello and G. Vitiello, Phys. Lett. A 363, 53 (2007);

A. Capolupo, S. Capozziello and G. Vitiello, in Proceed of DICE 2006, Piombino 2006, in print, arXiv:hep-th/0612035.

[26] C. Itzykson and J. B. Zuber, Quantum Field Theory, (McGraw-Hill, New York, 1980).

S. Schweber, An itroduction Relativistic Quantum Field Theory, (Harper and Row, 1961).

[27] V. Sahni, Lect. Notes Phys. 653, 141 (2004).

[28] S. Perlmutter et al., ApJ 517, 565 (1999); R.A. Knop et al., ApJ 598, 102 (2003).

[29] A.G. Riess et al., AJ 116, 1009 (1998); J.L. Tonry et al., ApJ 594, 1 (2003).

[30] P. de Bernardis et al., Nature 404, 955 (2000).

[31] R. Stompor et al., ApJ 561, L7 (2001).

[32] D.N. Spergel et al. ApJS 148, 175 (2003).

[33] A.G. Riess et al., ApJ 607, 665 (2004).

[34] V., Sahni, A. Starobinski, Int. J. Mod. Phys. D 9, 373 (2000).

[35] T. Padmanabhan, Phys. Rept. 380, 235 (2003).

[36] S. Capozziello, S. Nojiri, S. D. Odintsov and A. Troisi, Phys. Lett. B 639, 135 (2006)

[37] A. Kamenshchik, U. Moschella, V. Pasquier, Phys. Lett. B 511, 265 (2001).

[38] T. Padmanabhan, Phys. Rev. D 66, 021301 (2002).

[39] B.A. Bassett, M. Kunz, D. Parkinson, C. Ungarelli, Phys. Rev. D 68, 043504 (2003).

[40] V.F. Cardone, A. Troisi, S. Capozziello, Phys. Rev. D 69, 083517 (2004); S. Capozziello, V.F. Cardone, E. Elizalde, S. Nojiri, S.D. Odintsov, Phys.Rev.D 73, 043512 (2006).

[41] S. Capozziello, V.F. Cardone and A. Troisi Jou. Cosm. and Astrop. Phys. 08, 001 (2006).

[42] S. Capozziello and R. Garattini, Class. Quant. Grav. 24, 1627 (2007).

[43] K. Freese, M. Lewis, Phys. Lett. B 540, 1 (2002).

[44] G.R. Dvali, G. Gabadadze, M. Porrati, Phys. Lett. B 485, 208 (2000).

[45] S. Capozziello, Int. J. Mod. Phys. D 11, 483 (2002);

S.Nojiri and S.D. Odintsov, Phys. Rev. D 68, 123512 (2003);

Carroll, S.M., Duvvuri, V., Trodden, M., Turner, M.S., Phys. Rev. D 70, 043528 (2004);

G. Allemandi, A. Borowiec, M. Francaviglia, Phys. Rev. D 70, 103503 (2004). 
[46] S. Capozziello, V.F. Cardone, E. Piedipalumbo, M. Sereno, A. Troisi, Int. J. Mod. Phys. D 12, 381 (2003).

[47] S. Capozziello, V.F. Cardone, S. Carloni, A. Trosi, Int. J. Mod. Phys. D 12, 1969 (2003). 